\title{
Efficient Contour Integral-based Eigenvalue Computation Using an Iterative Linear Solver with Shift-Invert Preconditioning
}

\author{
Yasunori Futamura \\ University of Tsukuba \\ Tsukuba, Japan \\ futamura@cs.tsukuba.ac.jp
}

\author{
Tetsuya Sakurai \\ University of Tsukuba \\ Tsukuba, Japan \\ sakurai@cs.tsukuba.ac.jp
}

\begin{abstract}
Contour integral-based (CI) eigenvalue solvers are one of the efficient and robust approaches for sparse eigenvalue problems. They have attracted attention owing to their inherent parallelism. For implementing a CI eigensolver, the inner linear systems arising in the algorithm need to be solved using an efficient method. One widely-used method is to use a sparse direct linear solver provided by a well-established numerical library; it is numerically robust and presents good load balancing of parallel execution of the CI eigensolver. However, owing to high total computational and memory cost, the performance of the direct solver approach is suboptimal. In this study, we propose an alternative method that utilizes a block Krylov iterative linear solver and shift-invert preconditioning that can take advantage of the shift-invariance of the block Krylov subspace. Our approach adaptively sets a preconditioning parameter according to the number of parallel processes to reduce the iteration counts. Several numerical examples confirm that our method outperforms the direct solver approach.
\end{abstract}

\section{CCS CONCEPTS}

- Mathematics of computing $\rightarrow$ Computations on matrices; • Computing methodologies $\rightarrow$ Massively parallel algorithms.

\section{KEYWORDS}

sparse generalized eigenvalue problem, contour integral-based solver, block Krylov subspace method, shift-invert preconditioning

\section{ACM Reference Format:}

Yasunori Futamura and Tetsuya Sakurai. 2021. Efficient Contour Integralbased Eigenvalue Computation Using an Iterative Linear Solver with ShiftInvert Preconditioning. In The International Conference on High Performance Computing in Asia-Pacific Region (HPCAsia 2021), fanuary 20-22, 2021, Virtual Event, Republic of Korea. ACM, New York, NY, USA, 10 pages. https://doi.org/10.1145/3432261.3432269

Permission to make digital or hard copies of all or part of this work for personal or classroom use is granted without fee provided that copies are not made or distributed for profit or commercial advantage and that copies bear this notice and the full citation on the first page. Copyrights for components of this work owned by others than ACM must be honored. Abstracting with credit is permitted. To copy otherwise, or republish, to post on servers or to redistribute to lists, requires prior specific permission and/or a fee. Request permissions from permissions@acm.org.

HPCAsia 2021, January 20-22, 2021, Virtual Event, Republic of Korea

(c) 2021 Association for Computing Machinery.

ACM ISBN 978-1-4503-8842-9/21/01 ..\$15.00

https://doi.org/10.1145/3432261.3432269

\section{INTRODUCTION}

The large-scale sparse eigenvalue problem is one of the most timeconsuming part of scientific computing applications, such as electronic structure calculations and structural analysis. Contour integralbased (CI) eigenvalue solvers [14-16, 18, 26, 30] are one of the efficient and robust methods to deal with the eigenvalue problem. They have attracted attention owing to their inherent parallelism, and have been adopted on massively parallel computational environments [7, 13, 19, 23].

To implement the $\mathrm{CI}$ eigensolver, an efficient method should be used for solving some parameterized linear systems arising in the algorithm. One robust approach is to employ one of the sparse direct linear solvers that are implemented in well-established libraries such as PARSIDO [32] and MUMPS [4].

Such an approach is numerically robust and provides good load balancing of parallel execution of the CI eigensolver because its computational cost is the same for the parameters of the linear systems. Compared to (preconditioned) iterative solvers, the robustness of direct linear solvers is beneficial for end users; they do not have to tune the preconditioner of the solver for their application. Because of this feature, predefined interfaces to direct linear solvers are given by CI eigensolver libraries such as z-Pares [5] and FEAST [3].

However, because of its total computational cost, the performance of the direct solver approach is suboptimal. In addition, when the number of quadrature points is larger than the number of processes while we iterate the $\mathrm{CI}$ eigensolver, the factors of the (e.g. LU) factorization of multiple (complex-valued) coefficient matrices have to be stored on the main or secondary memory, which leads to serious increase of memory and I/O cost.

In this study, we propose an alternative approach that utilizes a block Krylov iterative linear solver and shift-invert preconditioning that can take advantage of the shift-invariance of the block Krylov subspace. Using the iterative solver with shift-invert preconditioning, only one coefficient matrix needs to be factorized to solve groups of linear systems. Meanwhile, with the shift-invariance of the subspace, we only have to perform a pair of forward and backward substitutions (FBS), once for each iteration. In addition, the factorization can be performed in real arithmetic. Our approach adaptively sets a parameter of preconditioning according to the number of processes given to the CI solver. As the number of process increases, the iteration counts of linear systems decrease.

Several numerical examples demonstrate that our approach outperforms the direct solver approach in most cases.

The outline of this paper is described below. In the next section, we present an algorithm of a contour integral-based eigensolver. In Section 3, we show an iterative linear solver based on the block 
Krylov subspace and shift-invert preconditioning. In Section 4, a parallel implementation of the entire algorithm of our approach is demonstrated. In Section 5, we illustrate the numerical experiments for the performance evaluation of our approach. The conclusion is given in Section 6.

\section{PARALLEL CONTOUR INTEGRAL-BASED EIGENVALUE SOLVER}

In this study, we consider solving generalized eigenvalue problems

$$
A \boldsymbol{u}=\lambda B \boldsymbol{u}
$$

where $A \in \mathbb{R}^{n \times n}$ is symmetric and $B \in \mathbb{R}^{n \times n}$ is symmetric positive definite. We assume that $A$ and $B$ are large and sparse. The scalar $\lambda$ is an eigenvalue while $\boldsymbol{x} \in \mathbb{R}^{n} \backslash\{0\}$ is an eigenvector. All eigenvalues of (1) are known to be real. The eigenvectors can be formed as $B$-orthonormal basis, i.e. $\boldsymbol{u}_{i}^{\mathrm{T}} B \boldsymbol{u}_{j}=\delta_{i, j}$, where $\delta_{i, j}$ is the Kronecker delta. We consider computing all eigenvalues in the interval $[a, b]$ and corresponding eigenvectors. Assume that the eigenvalues are not located on the boundary of the interval. The form of eigenvalue problem (1) has many applications, such as structural analysis and electronic structure calculations.

Here, we denote $I_{m}$ as the identity matrix of order $m$, and $O_{n \times m}$ as the $n \times m$-dimensional zero matrix. For a matrix $C, C^{\mathrm{T}}$ is the transpose and $C^{\mathrm{H}}=\bar{C}^{\mathrm{T}}$ is the conjugate-transpose.

\subsection{Sakurai-Sugiura Projection Method}

In [29], the so-called Sakurai-Sugiura method (SSM) was proposed for generalized eigenvalue problems with (in general) non-Hermitian matrices using contour integral. Block versions and Rayleigh-Ritztype (SS-RR), as well as Arnoldi-type variants have been proposed [14$16,18,30]$. The FEAST eigensolver [26] originated from the density matrix representation in quantum mechanics is also categorized as the contour integral-based solver.

Herein, we illustrate the SSM. The method is based on matrices defined by the contour integral:

$$
\mathcal{P}_{k}:=\frac{1}{2 \pi \mathrm{i}} \oint_{\Gamma} z^{k}(z B-A)^{-1} B \mathrm{~d} z \quad(k=0,1, \ldots) .
$$

$\Gamma$ is a positively oriented Jordan curve (on the complex plane) that intersects at $a$ and $b$ on the real axis.

Using the spectral decomposition of $B^{-1} A$ and Cauchy's integral expression, we have:

$$
\begin{aligned}
\mathcal{P}_{k} & =\frac{1}{2 \pi \mathrm{i}} \oint_{\Gamma} \sum_{i=1}^{n} \frac{z^{k}}{z-\lambda_{i}} \boldsymbol{u}_{i} \boldsymbol{u}_{i}^{\mathrm{T}} B \mathrm{~d} z \\
& =\sum_{i, \lambda_{i} \in[a, b]} \lambda_{i}^{k} \boldsymbol{u}_{i} \boldsymbol{u}_{i}^{\mathrm{T}} B
\end{aligned}
$$

Therefore, by applying $\mathcal{P}_{k}$ to a vector $\boldsymbol{v}_{0}$, we can extract the components of eigenvectors of interest from $\boldsymbol{v}_{0}$. Block (multi-vector) versions of the SSM $[14,15]$ consider applying $\mathcal{P}_{k}$ to $V_{0} \in \mathbb{R}^{n \times \ell}$ $(\ell \ll n) . V_{0}$ is referred to as the source (or input) matrix, which is normally generated by random numbers.
The contour integral is discretized using some quadrature rule with $N_{\mathrm{q}} \ll n$ quadrature points as:

$$
S_{k}:=\sum_{i=1}^{2 N_{\mathrm{q}}} w_{j} \zeta_{j}^{k}\left(z_{j} B-A\right)^{-1} B V_{0} \approx \mathcal{P}_{k} V_{0}
$$

with quadrature points $z_{j}$ and quadrature weights $w_{j} . \zeta_{j}$ are given by some normalization of the quadrature points for numerical stability [31]. In practice, the quadrature points are placed symmetrically with respect to the real-axis. Since $A, B$, and $V_{0}$ are real, with symmetrically placed quadrature points, $S_{k}$ can be further be written as:

$$
S_{k}=2 \sum_{i=1}^{N_{\mathrm{q}}} \operatorname{Re}\left(w_{j} \zeta_{j}^{k}\left(z_{j} B-A\right)^{-1}\right) B V_{0},
$$

where $\operatorname{Re}(\cdot)$ is the real-part. Figure 1 illustrates an example of numerical contour integration.



Figure 1: Numerical contour integration.

$$
\begin{aligned}
& \text { We define matrix } S \in \mathbb{R}^{n \times \ell M}\left(M \leq N_{\mathrm{q}}\right) \text { as: } \\
& \qquad S:=\left[S_{0}, S_{1}, \ldots, S_{M-1}\right] .
\end{aligned}
$$

When $M>1$, because of the term $\lambda_{i}^{k}$ in (3), multiple (approximated) linear combinations of eigenvectors are obtained.

There exist several approaches to extract eigenvectors from the range space of $S$. These approaches result in different variants of the SSM. The variant SS-RR $[14,30]$ uses the Rayleigh-Ritz procedure [27] that solves a small eigenvalue problem with matrices at most $\ell M$ dimension. The FEAST eigensolver is closely related to SS-RR with $M=0$.

When the accuracy of eigenpairs is not satisfactory, the numerical contour integration can be iterated. One iteration technique is to use linear combinations of the approximate eigenvectors as $V_{0}$ of the next iteration. For more details on the parameter setting and practical usage of the SSM, refer to [31].

The most time-consuming part of the SSM is the computation of $S_{k}$ in (5) because of the matrix inverse. This requires solutions of $N_{\mathrm{q}}$ linear systems parameterized with $z_{j}$ :

$$
\left(z_{j} B-A\right) X_{j}=V \quad \text { for } j=1,2, \ldots, N_{\mathrm{q}},
$$

where $V:=B V_{0}$. For each $j$, we have $\ell$ right-hand side vectors since $V$ has $\ell$ columns. We refer to a set of linear systems (with $\ell$ right-hand sides) for each $j$ as a block linear system.

When we place multiple contour paths in the region where the eigenvalues of interest is located, the computations of numerical 


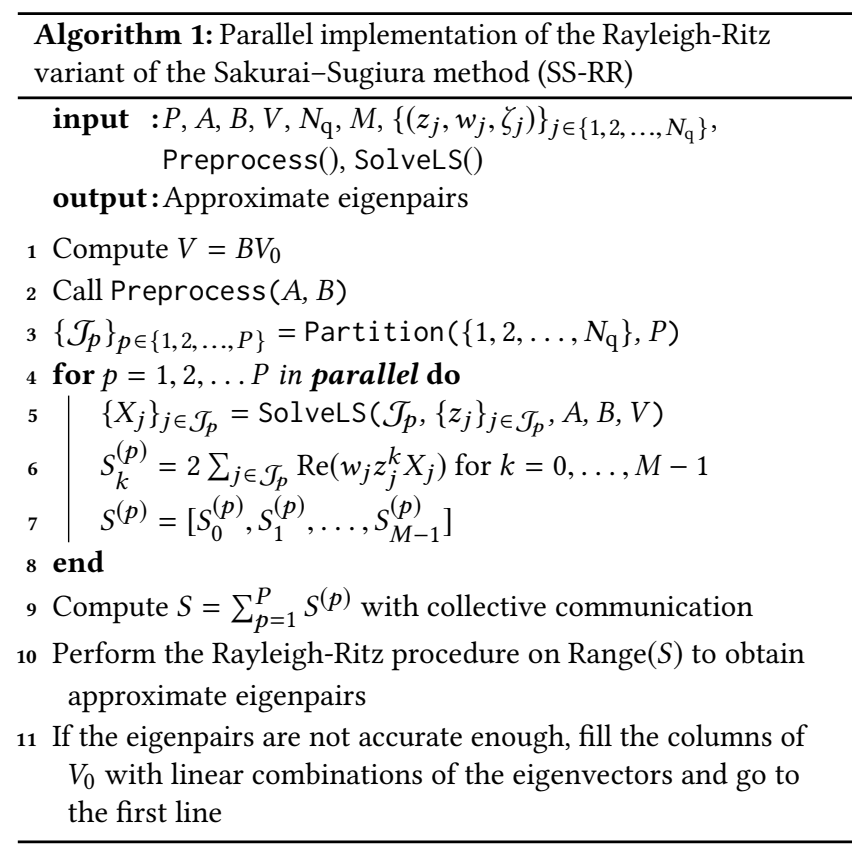

contour integrals are independent. For each contour path, we can solve the $N_{\mathrm{q}}$ block linear systems independently. Moreover, each block linear system is solved by a (distributed) parallel implementation of a linear system solver. This hierarchical parallelism of the $\mathrm{CI}$ eigensolvers can take advantage of massively parallel computational environments. Several studies apply the CI eigensolvers to large-scale practical applications on supercomputers [7, 13, 19, 23].

Here, we demonstrate the algorithm of the SSM (SS-RR) in Algorithm 1. In this study, we focus on parallelism for solving $N_{\mathrm{q}}$ block linear systems. Let $P$ be the number of parallel processes of distributed computing, and is no more than $N_{\mathrm{q}}$. We partition the index set $\left\{1,2, \ldots, N_{\mathrm{q}}\right\}$ into $P$ disjoint subsets $\mathcal{J}_{p}(p=1,2, \ldots, P)$ with natural order. For example, when $N_{\mathrm{q}}=8$ and $P=2$, we set $\mathcal{J}_{1}=\{1,2,3,4\}$ and $\mathcal{J}_{2}=\{5,6,7,8\}$. This simple partition operation appears as Partition() in Algorithm 1.

In Algorithm 1, we introduce two functions Preprocess and SolveLS as input for the algorithm. Preprocess indicates a preprocessing for the linear solver that can be executed before entering the parallel for section. SolveLS is the solution step of the linear solver that solves $\left(z_{j} B-A\right) X_{j}=V$ for $X_{j}\left(j \in \mathcal{J}_{p}\right)$. Concrete examples will be illustrated in Section 2.2 and 3.1.

Note that the steps outside of parallel for section can be executed with either one process through broadcast communication or through redundant computation. The implementation details of these steps (except the inside of Preprocess) are not relevant to our comparison in the performance evaluation.

Although we perform the numerical experiment using the SSM, our new approach can be applicable for other CI eigensolvers, Therefore, hereafter, we simply refer to the SSM as the CI eigensolver.

\subsection{Direct Solver Approach}

As discussed above, the $N_{\mathrm{q}}$ block linear systems (7) in the algorithm of the CI eigensolver need to be solved. One of the most numerically robust approaches for solving the linear systems is to use the direct method with the sparse matrix factorization. Using complex sparse LDL (modified Cholesky) factorization $L_{j} D_{j} L_{j}^{\mathrm{T}}=z_{j} B-A$ is reasonable because $z_{j} B-A$ are complex symmetric. The distributed and/or shared memory parallel direct linear solver routines are provided by well-established libraries, such as PARDISO and MUMPS.

As the computational cost mainly depends on the non-zero pattern of the matrix (in contrast to iterative methods), all block linear systems can be solved with almost the same amount of computation time when using a direct solver. This feature leads to good load balance for the CI eigensolver. Therefore, the CI solver libraries, such as z-Pares and FEAST, provide interface to direct solver libraries, which users can utilize in a plug-and-play manner.

Algorithm 2 illustrates the definitions of functions work as Preprocess and SolveLS. In DS_Preprocess, we perform analysis of the non-zero pattern of the coefficient matrix. This consists of fill-reducing ordering and symbolic factorization, and can be performed without numerical value of the matrix. In DS_SolveLS, using the result of the preprocessing phase, we sequentially perform the numerical factorization and FBS along with the index set $\mathcal{J}$.

However, the direct solver approach becomes expensive when a large number of fill-ins are generated. When we iterate the $\mathrm{CI}$ eigensolver and $P<N_{\mathrm{q}}$, the situation becomes more challenging. In such cases, the multiple LDL factors are required to be stored on the main or secondary memory, incurring I/O cost, which is not practical for large-scale problems. If we avoid storing the factors, numerical factorization (which is the most costly) should be performed in every CI iteration, which is expensive.

In this study, we propose a new alternative approach which basically uses an iterative linear solver. Although our approach also relies on the direct solver, the numerical factorization should be performed only once even if $|\mathcal{J}|>1(|\mathcal{J}|$ is the number of elements of the set).

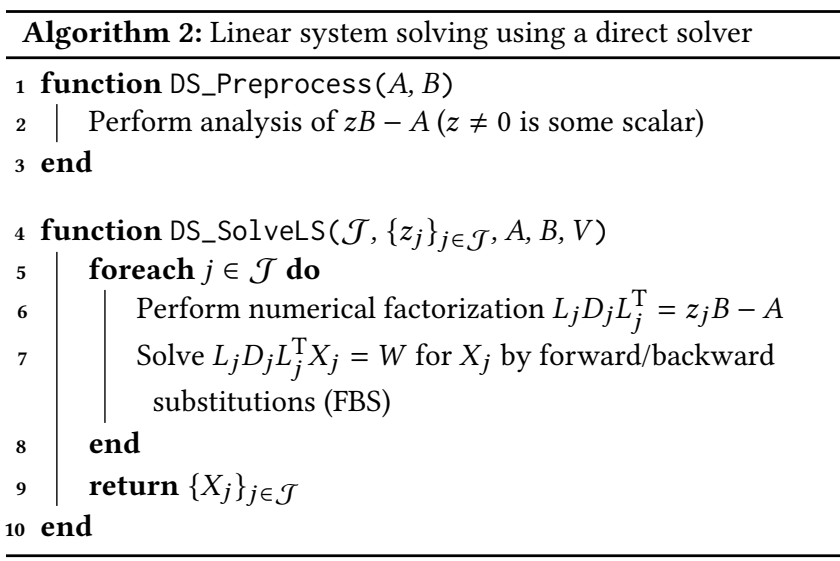

\section{BLOCK KRYLOV LINEAR SOLVER WITH SHIFT-INVERT PRECONDITIONING}

The block linear systems (7) can be considered parameterized linear systems with the quadrature points $z_{j}$. By utilizing this characteristic, we form an iterative linear solver based on shift-invert 
preconditioning that can take advantage of the shift-invariance of the block Krylov subspace.

\subsection{Shifted Block Minimum Residual Method with Shift-Invert Preconditioning}

During the algorithm derivation, we drop the subscripts of $z_{j}$ and $X_{j}$ for simplicity. We now consider a block linear system:

$$
(z B-A) X=V \text {. }
$$

For general block linear systems, block Krylov subspace methods, such as the block conjugate-gradient (CG) method [24] and the block generalized minimal residual (GMRES) method [35], are widely used. The block Krylov methods usually require fewer number of iteration than the standard (single-vector) Krylov methods due to larger search subspace. For general square matrix $C \in \mathbb{C}^{n \times n}$ and non-zero $V \in \mathbb{C}^{n \times \ell}$, the block Krylov subspace [11] is defined as:

$$
\mathcal{B}_{k}(C ; V):=\left\{\sum_{i=1}^{k} C^{i-1} V \xi_{i} \mid \xi_{i} \in \mathbb{C}^{\ell \times \ell}\right\}
$$

For some scalers $\alpha \neq 0 \in \mathbb{C}$ and $\beta \in \mathbb{C}$, it is known that:

$$
\mathcal{B}_{k}(\alpha C+\beta I ; V)=\mathcal{B}_{k}(C ; V),
$$

where $I:=I_{n}$. This is called the shift invariance of the subspace [34].

3.1.1 Shift-Invert Preconditioning. We introduce the preconditioner used in [20]. By multiplying $(\sigma B-A)^{-1}$ to the coefficient matrix of (8), we obtain:

$$
\begin{aligned}
& (\sigma B-A)^{-1}(z B-A) \\
& =(\sigma B-A)^{-1}((z-\sigma) B+\sigma B-A) \\
& =(z-\sigma)(\sigma B-A)^{-1} B+I .
\end{aligned}
$$

Here, we assume that $\sigma \in \mathbb{R}$ does not equal any of eigenvalues of (1), so that $(\sigma B-A)$ is invertible. By using (11), (8) can be rewritten as:

$$
\left((z-\sigma)(\sigma B-A)^{-1} B+I\right) X=(\sigma B-A)^{-1} V .
$$

By defining $G_{\sigma}$ and $F_{\sigma}(z)$ as:

$$
G_{\sigma}:=(\sigma B-A)^{-1} B
$$

and

$$
F_{\sigma}(z):=(z-\sigma) G_{\sigma}+I,
$$

and setting $\widehat{V}:=(\sigma B-A)^{-1} V$, (12) can be further expressed as:

$$
\left((z-\sigma) G_{\sigma}+I\right) X=\widehat{V} .
$$

We refer to this transformation (preconditioning) as the shift-invert preconditioning. We call $\sigma$ the shift parameter. By the shift invariance of the block Krylov subspace, we have:

$$
\mathcal{B}_{k}\left(F_{\sigma}(z) ; \widehat{V}\right)=\mathcal{B}_{k}\left(G_{\sigma} ; \widehat{V}\right) \text {. }
$$

3.1.2 Block Lanczos Procedure. We utilize the block Lanczos procedure for $G_{\sigma}$, which was introduced in [10], that can form $B$ orthonormal basis of the block Krylov subspace. Algorithm 3 shows the block Lanczos procedure. In the algorithm, the function $Q, R=$ WQR $(V, B)$ with $V \in \mathbb{R}^{n \times \ell}$ and symmetric positive definite matrix $B \in \mathbb{R}^{n \times n}$ indicates the matrix-weighted (thin) $\mathrm{QR}$ decomposition of $V$. That is, it is the operation computing $Q \in \mathbb{R}^{n \times \ell}$ and $R \in \mathbb{R}^{\ell \times \ell}$, such that $Q^{\mathrm{T}} B Q=I_{\ell}, Q R=V$, while $R$ is upper triangular.

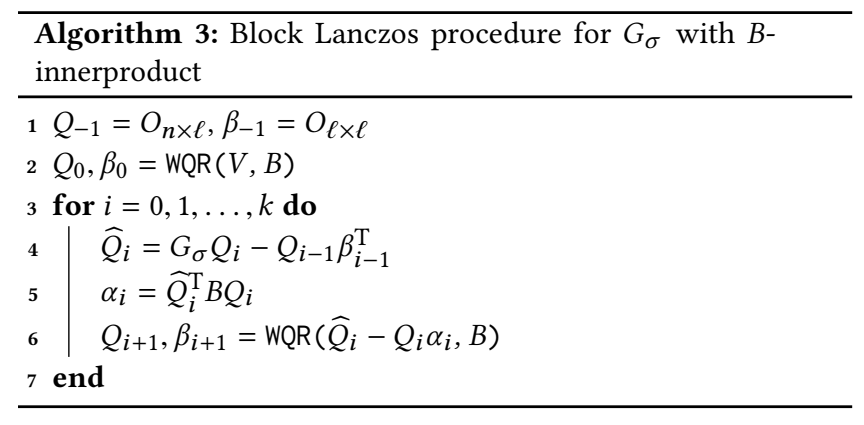

Algorithm 3 forms the block Lanczos decomposition:

$$
G_{\sigma} Q_{k}=Q_{k+1} \underline{\widehat{H}}_{k}
$$

where $Q_{k+1}:=\left[Q_{0}, Q_{1}, \ldots, Q_{k}\right] \in \mathbb{R}^{n \times(k+1) s}$, and $\widehat{\widehat{H}}_{k} \in \mathbb{R}^{(k+1) \ell \times k \ell}$ indicates the banded matrix with $\ell$ subdiagonal elements $\left(Q_{k}\right.$ is defined respectively). The structure of $\underline{\hat{H}}_{k}$ is shown as:

$$
\underline{\widehat{H}}_{k}=\left(\begin{array}{ccccc}
\alpha_{0} & \beta_{1}^{\mathrm{T}} & & & \\
\beta_{1} & \alpha_{1} & \beta_{2}^{\mathrm{T}} & & \\
& \beta_{2} & \ddots & \ddots & \\
& & \ddots & & \beta_{k-1}^{\mathrm{T}} \\
& & & & \alpha_{k-1} \\
& & & & \beta_{k}
\end{array}\right) .
$$

It is known that $Q_{k}^{\mathrm{T}} B Q_{k}=I_{k \ell}$ and $Q_{k}^{\mathrm{T}} B G_{\sigma} Q_{k}=\widehat{H}_{k}$, where $\widehat{H}_{k}$ is the top submatrix of $\underline{\underline{H}}_{k}$ with $k \ell$ rows.

Note that, though $G_{\sigma}$ is not symmetric, under the $B$-innerproduct $\left(\boldsymbol{x}^{\mathrm{H}} B \boldsymbol{y}\right.$ with vectors $\boldsymbol{x}$ and $\left.\boldsymbol{y}\right)$, this block Lanczos procedure can form the $B$-orthogonal basis by a short-term recurrence. Also note that, $\sigma$ should be real to ensure this feature.

By the shift invariance of the block Krylov subspace, $Q_{k}$ also forms basis of $\mathcal{B}_{k}\left(F_{\sigma}(z) ; \widehat{V}\right)$. The block Lanczos decomposition of $F_{\sigma}(z)$ is given as:

$$
F_{\sigma}(z) Q_{k}=Q_{k+1} \underline{H}_{k}(z)
$$

where

$$
\underline{H}_{k}(z):=(z-\sigma) \underline{\hat{H}}_{k}+\left[I_{\ell}, O_{\ell \times k \ell}^{\mathrm{T}}\right]^{\mathrm{T}} .
$$

3.1.3 Minimum residual condition. Based on the block Lanczos decomposition, we introduce an algorithm for solving (8) with minimum residual condition by following the derivation of the block MINRES method [33] (which was proposed for general symmetric indefinite matrices with the standard inner product).

Let the (standard full) QR decomposition of $\underline{H}_{k}(z)$ be:

$$
\Theta_{k} \underline{R}_{k}=\underline{H}_{k}(z) \text {. }
$$

Here, $\Theta_{k} \in \mathbb{C}^{(k+1) \ell \times(k+1) \ell}, \underline{R}_{k} \in \mathbb{C}^{(k+1) \ell \times k \ell}$, and $\Theta_{k}\left(\Theta_{k}\right)^{\mathrm{H}}=$ $I_{(k+1) \ell}$, while $\underline{R}_{k}$ is the upper triangular. $\underline{R}_{k}$ is actually an upper banded matrix with at most $2 \ell+1$ non-zero entries for each row. We write the structure of $\underline{R}_{k}$ as:

$$
\underline{R}_{k}=\left[R_{k}^{\mathrm{H}}, O_{\ell \times k \ell}^{\mathrm{H}}\right]^{\mathrm{H}}
$$


with

$$
R_{k}:=\left(\begin{array}{cccccc}
\rho_{1} & \widehat{\phi}_{1} & \widehat{\psi}_{1} & & & \\
& \rho_{2} & \widehat{\phi}_{2} & \widehat{\psi}_{2} & & \\
& & \ddots & \ddots & \ddots & \\
& & & & & \widehat{\psi}_{k-2} \\
& & & & & \widehat{\phi}_{k-1} \\
& & & & & \rho_{k}
\end{array}\right) \in \mathbb{C}^{k \ell \times k \ell} .
$$

Here, $\rho_{i}, \widehat{\phi}_{i}$, and $\widehat{\psi}_{i}$ are $\ell \times \ell$ matrices. Note that $\rho_{i}$ and $\widehat{\psi}_{i}$ are upper and lower triangular, respectively. $\underline{R}_{k}$ and $\Theta_{k}$ can be iteratively formed from $\underline{H}_{k}(z)$ by Householder transformations [9] involving $2 \ell+1$ rows at each iteration of the algorithm shown later.

We introduce $B$-weighted Frobenius norm as:

$$
\|X\|_{B}=\sqrt{\operatorname{tr}\left(X^{\mathrm{H}} B X\right)} .
$$

When $B=I$, this is equivalent to the standard Frobenius norm, i.e. $\|X\|_{I}=\|X\|_{\mathrm{F}}$.

Next, we consider minimizing the $B$-weighted Frobenius norm of the residual of (15) with $X \in \mathcal{B}_{k}\left(G_{\sigma} ; \widehat{V}\right)$. The minimizing condition is:

$$
\min _{X \in \mathcal{B}_{k}\left(G_{\sigma} ; \widehat{V}\right)}\left\|\widehat{V}-G_{\sigma} X\right\|_{B}=\min _{Y \in \mathbb{C}^{k \ell \times \ell}}\left\|\widehat{V}-G_{\sigma} Q_{k} Y\right\|_{B} .
$$

The norm in the right-hand side is:

$$
\begin{aligned}
\left\|\widehat{V}-G_{\sigma} Q_{k} Y\right\|_{B} & =\left\|Q_{0} \beta_{0}-Q_{k+1} \underline{H}_{k} Y\right\|_{B} \\
& =\left\|Q_{k+1} E_{k} \beta_{0}-Q_{k+1} \underline{H}_{k} Y\right\|_{B} \\
& =\left\|E_{k} \beta_{0}-\underline{H}_{k} Y\right\|_{\mathrm{F}} \\
& =\left\|\Theta_{k}^{\mathrm{H}} E_{k} \beta_{0}-\underline{R}_{k} Y\right\|_{\mathrm{F}} .
\end{aligned}
$$

Here, $E_{k}$ is the left-most submatrix with $\ell$ columns of $I_{(k+1)} \ell$. Let

$$
\left[\widehat{W}_{0}, \widehat{W}_{1}, \ldots, \widehat{W}_{k-1}\right]:=Q_{k} R_{k} \in \mathbb{C}^{n \times k \ell}
$$

with $\widehat{W}_{i} \in \mathbb{C}^{n \times \ell}$ and:

$$
\left[\xi_{0}^{\mathrm{H}}, \xi_{1}^{\mathrm{H}}, \ldots, \xi_{k}^{\mathrm{H}}\right]^{\mathrm{H}}:=\Theta_{k}^{\mathrm{H}} E_{k} \beta_{0} \in \mathbb{C}^{(k+1) \ell \times \ell}
$$

with $\xi_{i} \in \mathbb{C}^{\ell \times \ell}$. Using these, $Y$ that satisfy (25) is given as:

$$
Y=R_{k}^{-1}\left[\xi_{0}^{\mathrm{H}}, \xi_{1}^{\mathrm{H}}, \ldots, \xi_{k-1}^{\mathrm{H}}\right]^{\mathrm{H}} .
$$

As a result, $X$ is

$$
X=Q_{k} Y=\left[\widehat{W}_{0}, \widehat{W}_{1}, \ldots, \widehat{W}_{k-1}\right]\left[\xi_{0}^{\mathrm{H}}, \xi_{1}^{\mathrm{H}}, \ldots, \xi_{k-1}^{\mathrm{H}}\right]^{\mathrm{H}} .
$$

By substituting (28) and (29) into (26) and using the fact that the bottom $\ell$ rows of $\widehat{R}_{k}$ are zero, we can find that the residual norm is given by $\left\|\xi_{k}\right\|_{\mathrm{F}}$. This allows us to compute the residual norm with less cost, and used it to evaluate the solution accuracy.

By taking advantage of the upper banded structure of $R_{k}, X$ and $\widehat{W}_{k}$ can be updated using short-term recurrences

$$
\widehat{W}_{k+1}=\left(Q_{k-1}-\widehat{W}_{k-1} \widehat{\psi}_{k-1}-\widehat{W}_{k} \widehat{\phi}_{k}\right)\left(\rho_{k+1}\right)^{-1}
$$

and

$$
X_{k+1}=X_{k}+\widehat{W}_{k+1} \xi_{k+1} .
$$

The resulting algorithm is shown in Algorithm 4. We introduce

$$
W_{k}:=\widehat{W}_{k} \rho_{k}, \phi_{k}:=\rho_{k}^{-1} \widehat{\phi}_{k}, \psi_{k}:=\rho_{k}^{-1} \widehat{\psi}_{k}
$$

to reduce the dense matrix multiplications involving $n \times \ell$ matrices. In our study, the WQR operation is performed by the CholeskyQR2 method [36].

We refer to the algorithm as the shift-invert preconditioned shifted block MINRES method (SISB-MINRES). In SISB-MINRES, we only need one LDL factorization to solve all linear systems associated with $\left\{z_{j}\right\}_{j \in \mathcal{J}}$. Therefore, in contrast to the direct solver approach, it is possible to perform not only the analysis of the matrix but also the numerical factorization in Preprocess. Additionally, the factorization (and also FBS) can be performed with real arithmetic because $\sigma B-A$ is real. Furthermore, by making use of the shift-invariance of the block Krylov subspace, the FBS (operation of the preconditioner) of a set of $\ell$ vectors is performed only once at each iteration to update the solutions of $|\mathcal{J}|$ block linear systems.

3.1.4 Remark. The standard (non-block) MINRES method [25] with shift-invert preconditioning for the same kind of linear systems as (7) was proposed and analyzed by Meerbergen [20] for single-vector case. Similarly, for multiple-vector case, Meerbergen et al. [21] proposed an extended method, which is not based on the block Krylov subspace, but a deflation technique.

The block MINRES method was presented by Soodhalter [33] for general symmetric indefinite matrices with standard innerproduct. Their block MINRES updates the solution in column wise manner to cope with possible (near) linear independence of the column vectors. In our case, as we need to update the solutions and the related matrices $\left(X_{k}^{(j)}\right.$ and $\left.W_{k}^{(j)}\right)|\mathcal{J}|$-times in each iteration, we prefer and adopt the block-wise update that can utilize Level-3 BLAS operations. Implementing a treatment for possible numerical instability due to near linear independence is left for future work.

The block Lanczos procedure for matrices such as $G_{\sigma}$ was presented by Grimes et al. [10]. Though $G_{\sigma}$ is not symmetric, under the $B$-innerproduct, a certain symmetry can be utilized to form the short-term recurrence.

The newly presented SISB-MINRES of this study is derived by a combination of the ideas from the abovementioned literatures [ 10 , $20,33]$.

General preconditioning techniques, such as the incomplete LU factorization, can be applied to the linear systems (7). Iterative methods like (block) GMRES [17, 28, 35] with one of those preconditioners could be efficient if they are tuned for the application. In such cases, the operation of $(\sigma B-A)^{-1}$ in SISB-MINRES can also be performed through such preconditioned iterative method.

Although performance comparison involving general preconditioning techniques is interesting, in this study, we consider comparing the approaches based on a direct solver because it has few application specific matters.

3.1.5 Selecting shift parameter. The shift parameter $\sigma$ governs the convergence rate of SISB-MINRES. When $\sigma$ is close to $z$, the convergence speed is likely to be high as the preconditioned matrix $(\sigma B-A)^{-1}(z B-A)$ is better conditioned. However, since we apply SISB-MINRES to linear systems with multiple $z$, we should find a good location to put $\sigma$ to balance the convergence speed. While the optimal location may be determined by the eigenvalues of (1), we cannot use them in practice. 
As a simple heuristic, we determine $\sigma$ as:

$$
\sigma=\frac{1}{|\mathcal{J}|} \sum_{j \in \mathcal{J}} \operatorname{Re}\left(z_{j}\right) .
$$

Using this formula, we intend to place $\sigma$ at the median point of $\left\{z_{j}\right\}_{j \in \mathcal{J}}$, but take the real part because of the real-value constraint.

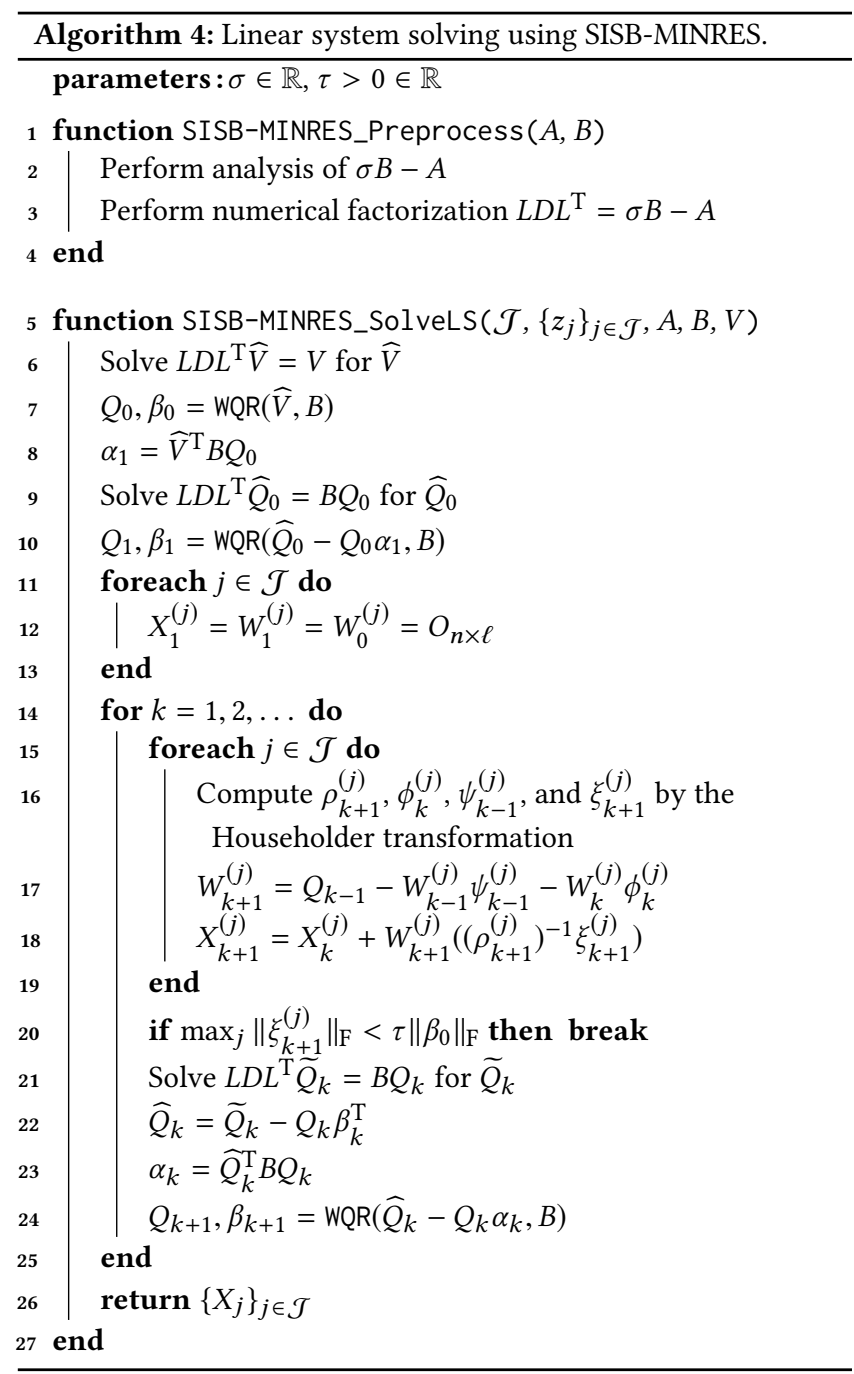

Table 1: Counts of main computational kernels for solving block linear systems with $N_{\mathrm{q}} / P$ coefficient matrices.

\begin{tabular}{lrrr}
\hline Kernel & Direct & SISB-MINRES & Time \\
\hline Real LDL & - & 1 & $T_{\text {r.fact }}$ \\
Complex LDL & $N_{\mathrm{q}} / P$ & - & $T_{\text {c.fact }}$ \\
Real FBS & - & Iter & $T_{\text {r.fbs }}$ \\
Complex FBS & $N_{\mathrm{q}} / P$ & - & $T_{\text {c.fbs }}$ \\
Complex BAXPY & - & $3\left(N_{\mathrm{q}} / P\right) \cdot$ Iter & $T_{\text {baxpy }}$ \\
\hline
\end{tabular}

\section{PARALLEL IMPLEMENTATION}

In this section, the parallel implementation of the CI eigensolver using multiple instances of SISB-MINRES has been discussed. The computational complexity of the direct solver approach and the proposed new SISB-MINRES approach have been compared.

\subsection{Adaptive Setting of Shift Parameters}

For the parallel execution of the $\mathrm{CI}$ eigensolver with $P$ processes, we partition the index set $\left\{1,2, \ldots, N_{\mathrm{q}}\right\}$ into $\left\{\mathcal{J}_{p}\right\}_{p=1}^{P}$. Therefore, when we utilize SISB-MINRES, $P$ instances of the method are simultaneously applied for $P$ sets of block linear systems.

For simplicity, we assume that $N_{\mathrm{q}}$ is divisible by $P$. For load balancing, we simply partition as $\left|\mathcal{J}_{p}^{(P)}\right|=N_{\mathrm{q}} / P$ for all $p$ (superscript $P$ is added to explicitly notify that it depends on $P$ ). By following the rule (34) determining $\sigma, \sigma_{p}$ (of $p$-th process) are different and depend on $p$. Moreover, $\sigma_{p}$ depend also on $P$, which should be distinguished as $\sigma_{p}^{(P)}$.

When we partition $\left\{1,2, \ldots, N_{\mathrm{q}}\right\}$ such that $\left\{z_{j}\right\}_{j \in\left\{1,2, \ldots, N_{\mathrm{q}}\right\}}$ are clustered, as we increase $P$, on average, $\sigma_{p}^{(P)}$ are closer to $\left\{z_{j}\right\}_{j \in \mathcal{J}_{p}^{(P)}}$. Thus, as $P$ increases, the iteration counts of SISB-MINRES is expected to decrease. As a result, the total sequential computational cost depends on $P$.

In Figure 2, we illustrate some examples of locations of $\sigma_{p}$.
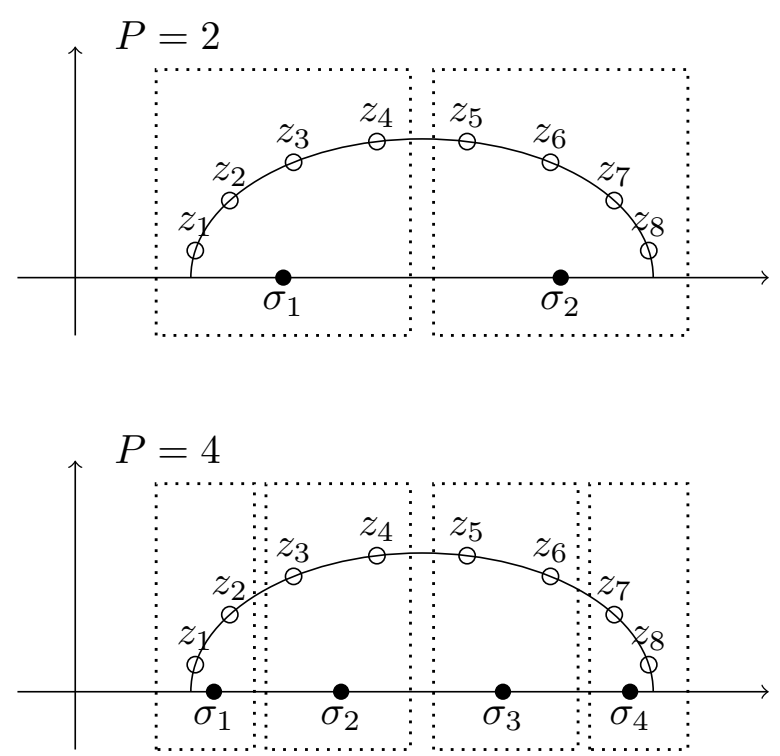

Figure 2: Adaptive setting of shift parameter $\sigma$.

\subsection{Computational Time Comparison}

In Table 1, we list the counts of main computational kernels of both the direct solver and the SISB-MINRES approaches required for an individual parallel process. LDL indicates the LDL factorization. FBS means FBS for $\ell$ vectors. Each symbol in the Time column 
means the computational time for single execution of the kernel. Iter is the iteration count of SISB-MINRES on the process.

BAXPY stands for Block AXPY (AXPY comes from a routine name of BLAS), which indicates the operation:

$$
Y \leftarrow Y+X \alpha,
$$

where $X, Y \in \mathbb{C}^{n \times \ell}$ and $\alpha \in \mathbb{C}^{\ell \times \ell}$. It is implemented with the ZGEMM routine of BLAS. We treat this as a main kernel because the computation of $X_{k+1}^{(j)}$ and $W_{k+1}^{(j)}$ (using BAXPY) is not negligible when $|\mathcal{J}|=N_{\mathrm{q}} / P$ is large and FBS is relatively inexpensive.

The parallel computational time of the direct solver approach is simply written as:

$$
T_{\text {direct }}(P):=\left(T_{\text {c.fact }}+T_{\text {c.fbs }}\right)\left(N_{\mathrm{q}} / P\right) .
$$

Owing to the difference of iteration counts, the computational time of the SISB-MINRES approach is complicated. When $P$ processes are used, the computational time on the $p$-th process can be represented as:

$$
T_{\text {sisb }}^{(P, p)}:=T_{\text {r.fact }}+\left[T_{\text {r.fbs }}+3\left(N_{\mathrm{q}} / P\right) T_{\text {baxpy }}\right] \cdot \operatorname{Iter}\left(\sigma_{p}^{(P)}\right) .
$$

Here, $\operatorname{Iter}\left(\sigma_{p}^{(P)}\right)$ is the iteration count of SISB-MINRES with $\sigma_{p}^{(P)}$ for solving all systems associated with $\left\{z_{j}\right\}_{j \in \mathcal{J}_{p}^{(P)}}$. The term $3\left(N_{\mathrm{q}} / P\right)$ is because three BAXPYs need to be performed for lines 17 and 18 of Algorithm 4 in each inner loop.

In $P$-parallel execution of instances of SISB-MINRES, we need to wait for the slowest process. The parallel computational time is:

$$
\begin{aligned}
T_{\text {sisb }}(P) & :=\max _{p \in\{1,2, \ldots, P\}} T_{\text {sisb }}^{(P, p)} \\
& =T_{\text {r.fact }}+\left[T_{\text {r.fbs }}+3\left(N_{\mathrm{q}} / P\right) T_{\text {baxpy }}\right] \cdot \operatorname{IterMax}(P)
\end{aligned}
$$

where

$$
\operatorname{IterMax}(P):=\max _{p \in\{1,2, \ldots, P\}} \operatorname{Iter}\left(\sigma_{p}^{(P)}\right) .
$$

Therefore, the parallel performance of our approach is determined by the balance of the iteration counts.

In both approaches, one factorization and at least one FBS should be performed when $P=N_{\mathrm{q}}$. Therefore, the computational times of the approaches are bounded by one pair of (real/complex) factorization and FBS. If further parallel scalability is expected, a distributed parallel linear solver needs to be used for the factorization and FBS.

\section{PERFORMANCE EVALUATION}

In this section, we present the performance comparison of the proposed approach and the direct solver approach.

\subsection{Experiment Setting}

We used four test problems listed in Table 2. In the table, nnz $(A)$ and $n n z(B)$ indicate the numbers of non-zero elements of $A$ and $B$, respectively. $\operatorname{nnz}(L)$ is the number of non-zero elements of the lower triangular factor of the LDL factorization of $z B-A$ reported by the analysis routine of PARDISO. $[a, b]$ is the interval for computing the eigenvalues. \# eigs is the number of eigenvalues in $[a, b]$. NCCS430080 was obtained from the ELSES matrix library $[2,12]$ bone 010 and Ga41As41H72 were obtained from the SuiteSparse matrix collection (formally University of Florida sparse matrix collection) [6]. CQSZ200K was obtained from a linear scaling DFT code CONQUEST $[1,8,22]$. The test problems were ordered by $n n z(L)$ which roughly indicates the computational cost to solve the problem. For CQSZ200K, the interval $[a, b]$ includes the eigenvalue associated with the highest occupied molecular orbital (HOMO). For other problems, the intervals include the smallest eigenvalues.

The program code was implemented in the Julia language version 1.3. We used non-builtin Julia packages related to numerical computing MKLSparse.jl, MPI.jl, and Pardiso.jl. All floating-point arithmetic were performed in double precision.

As the computational environment, we used the Oakforest-PACS supercomputer. A node of Oakforest-PACS equips one Intel Xeon Phi 7250 (67 cores) and DDR4-2400 96 GB memory.

As a (multi-threaded) direct linear solver, we used PARSIDO in Intel Math Kernel Library (MKL) through Pardiso.jl. Sparse matrix multiplications were performed with a routine of Intel MKL SparseBLAS with MKLSparse.jl. Dense matrix operations were performed using BLAS/LAPACK of Intel MKL.

We assigned single MPI process to a computational node, and thus, the number of processes indicates the number of nodes in our evaluation. For the intra-node parallelism, we utilized the multithreading capability of PARDISO, SparseBLAS, and BLAS/LAPACK. For all cases, we used 64 threads since the performance of the direct linear solver did not saturate up to that amount of threads in our preliminary experiments.

For the parameter setting of the CI eigensolver, we used $N_{\mathrm{q}}=16$, $M=8$, and $\ell=64$. The contour path was a flat ellipse with an aspect ratio of 0.1 [31]. The trapezoidal rule was used for numerical quadrature. We set the stopping criterion to be $\tau=1 \mathrm{e}-14$ for SISBMINRES. We did not iterate the CI eigensolver. Since $N_{\mathrm{q}}=16$, we used $P=1,2,4,8,16$ processes for the evaluation.

\subsection{Results}

In Table 3, we present the computational time of performing single real/complex factorization and FBS. Number of right hand sides for FBS is $\ell=64$. r.fact and c.fact are the time for the real and complex factorization. r.fbs and c.fbs are time for the real and complex FBS. r.ratio and c.ratio are the ratios r.fact/r.fbs and c.fact/c.fbs, respectively. We find that both real and complex cases, the ratio is the smallest for NCCS430080 and the largest for Ga41As41H72. This result will be used in later discussion.

In Table 4, we illustrate the maximum (and minimum in brackets) iteration counts among $P$-instances of SISB-MINRES. Since only one instance of SISB-MINRES is used when $P=1$, there exists no bracketed entry. For all cases, as $P$ increase, the iteration counts decrease as expected (see Section 4.1), while the reduction saturates when $P=16$. The difference of the maximum and minimum iteration counts directly affect the load balancing.

In Figure 3, 4, 5, and 6, we show the parallel computational time comparison of the problems NCCS430080, bone010, Ga41As41H72, and CQSZ200K, respectively. We exclude the multiplication of $B$ and the Rayleigh-Ritz procedure in lines 1 and 10 of Algorithm 1 from the computational time because they provide no impact on the comparison. For the same reason, we also excluded the computational time for the analysis of the matrix performed in line 2 of Algorithm 2 and line 2 of Algorithm 4. For each number of process, the left (blue) and right (orange) bars indicate the direct solver approach and our approach. 
Table 2: Properties of the matrices used in the numerical examples.

\begin{tabular}{lrrrrrr}
\hline Name & Size & $\operatorname{nnz}(A)$ & $\mathrm{nnz}(B)$ & $\mathrm{nnz}(L)$ & {$[a, b]$} & \# eigs \\
\hline NCCS430080 & 430,080 & $20,962,752$ & $20,962,752$ & $393,170,791$ & {$[-1.2,-1.181]$} & 201 \\
bone010 & 986,703 & $47,851,783$ & $B=I$ & $1,182,851,844$ & {$[1.0,45.0]$} & 211 \\
Ga41As41H72 & 268,096 & $18,488,476$ & $B=I$ & $2,398,280,235$ & {$[-1.3,-0.25]$} & 200 \\
CQSZ200K & 778,292 & $115,091,934$ & $85,258,344$ & $3,328,883,858$ & {$[0.0320,0.0329]$} & 216 \\
\hline
\end{tabular}

Table 3: Computational time [sec] for main kernels.

\begin{tabular}{lrrrrrr}
\hline & c.fact & c.fbs & c.ratio & r.fact & r.fbs & r.ratio \\
\hline NCCS430080 & 15.3 & 4.2 & 3.7 & 7.3 & 2.4 & 3.0 \\
bone010 & 65.9 & 14.0 & 4.7 & 30.8 & 6.3 & 4.9 \\
Ga41As41H72 & 534.6 & 48.7 & 11.0 & 219.6 & 16.0 & 13.7 \\
CQSZ200K & 358.1 & 56.2 & 6.4 & 152.9 & 19.7 & 7.8 \\
\hline
\end{tabular}

Table 4: Iteration counts for SISB-MINRES.

\begin{tabular}{lrrrrr}
\hline$P$ & 1 & 2 & 4 & 8 & 16 \\
\hline NCCS430080 & 41 & $30(18)$ & $19(11)$ & $15(8)$ & $14(7)$ \\
bone010 & 27 & $20(18)$ & $15(12)$ & $13(10)$ & $12(8)$ \\
Ga41As41H72 & 34 & $26(20)$ & $19(11)$ & $15(8)$ & $13(7)$ \\
CQSZ200K & 37 & $25(24)$ & $19(14)$ & $14(11)$ & $13(8)$ \\
\hline
\end{tabular}

For NCCS430080, when $P \leq 4$, our SISB-MINRES approach is faster than the direct solver approach. However, when $P=8,16$, the direct solver approach is faster. For bone010, when $P \leq 8$, our SISB-MINRES approach is faster, whereas when $P=16$, the direct solver approach is faster. For Ga41As41H72, our approach outperforms the other in all cases. For CQSZ200K, our approach outperforms the other when $P \leq 8$, whereas when $P=16$ the performances are almost similar. We observed that except NCCS430080, the performance gain is significant especially for $P \leq 4$. Note that, although the direct solver approach is faster in some cases, the memory cost of our approach is substantially less since it uses real-valued factorization.

For two particular examples NCCS430080 (the direct solver approach is faster when $P$ is large) and Ga41As41H72 (our approach, outperforms the other for all cases), the breakdowns of the elapsed times in Figure 7 and 8 have been demonstrated. For each number of process, the left and right bars indicate the direct solver approach and our approach, respectively. c.fact, c.fbs, and misc 1 are the times of factorization, FBS, and miscellaneous parts of the direct solver approach, respectively. r.fact, r.fbs, baxpy, and misc2 are the times of factorization, FBS, BAXPY, and miscellaneous parts of our approach, respectively.

We notice that the fractions of the factorization for Ga41As $41 \mathrm{H} 72$ is higher than those of NCCS430080. This result is associated with the ratios shown in Table 3. Ga41As41H72 is the highest and NCCS430080 is the lowest. Our approach is likely to be faster when the ratio is high, unless the number of iterations is too large.

While FBS dominates the performance, $P$-dependency of total FBS computing time in our approach comes from IterMax $(P)$

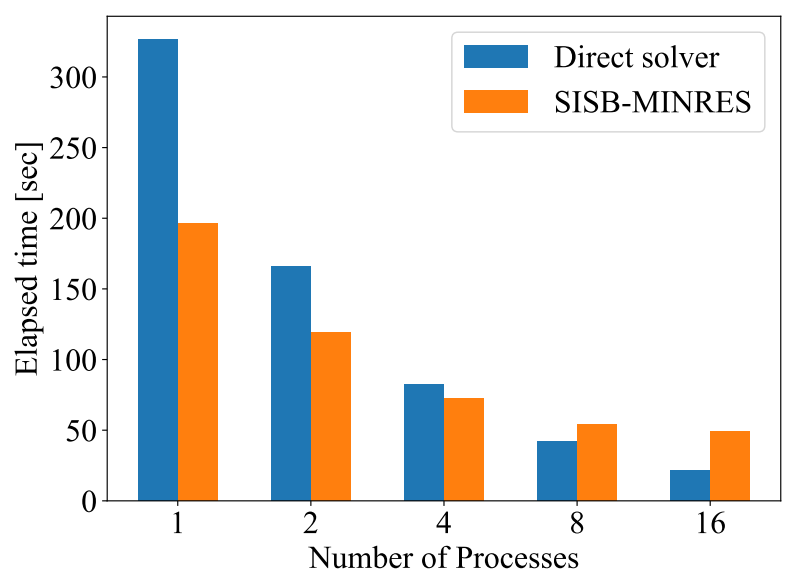

Figure 3: Elapsed time comparison for NCCS430080.

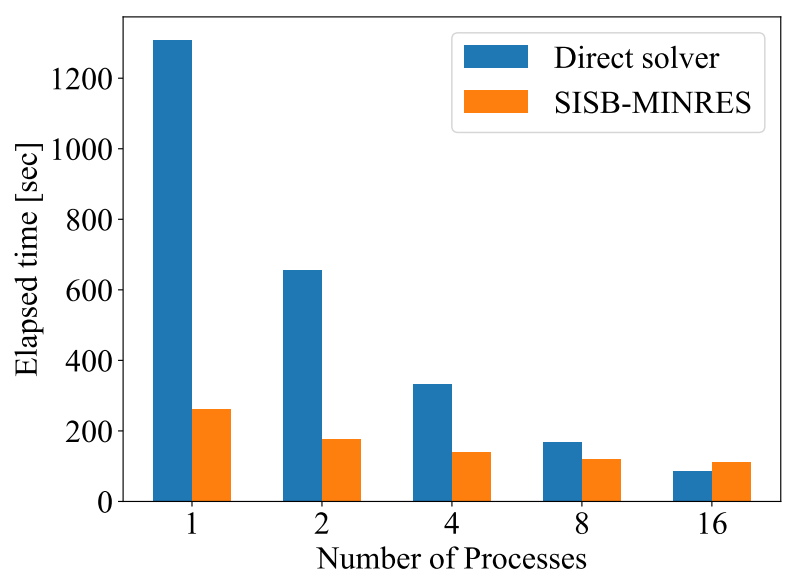

Figure 4: Elapsed time comparison for bone 010.

(see (38)). Therefore, the parallel performance of our approach mainly results from the reduction of the iteration counts.

Note that since we set $N_{\mathrm{q}}=16$, in both approaches, $P=16$ is the upper limit for the parallelism of the CI solver we used here. As mentioned in Section 4.2, if further parallel scalability is expected, a distributed parallel direct linear solver can be employed.

In Table 5, to assess the resulting accuracies of the eigenpairs computed by the CI eigensolver (with both approaches), we show 


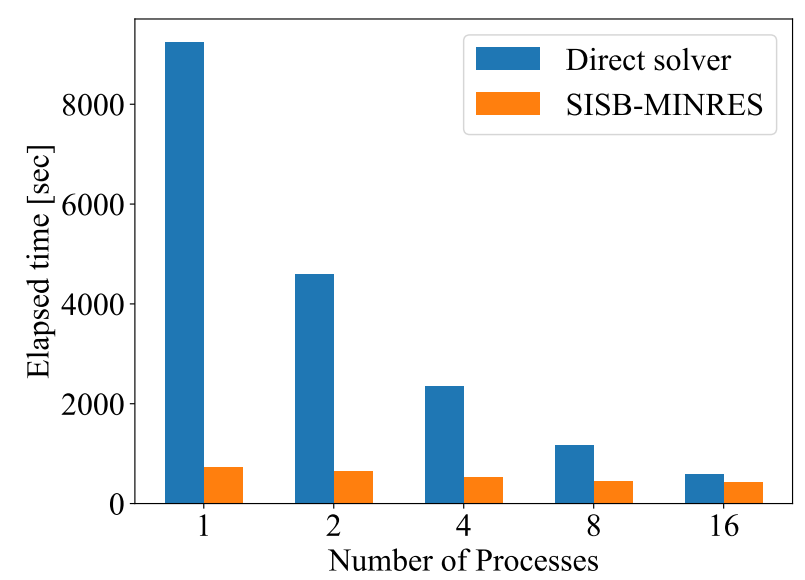

Figure 5: Elapsed time comparison for Ga41As41H72.

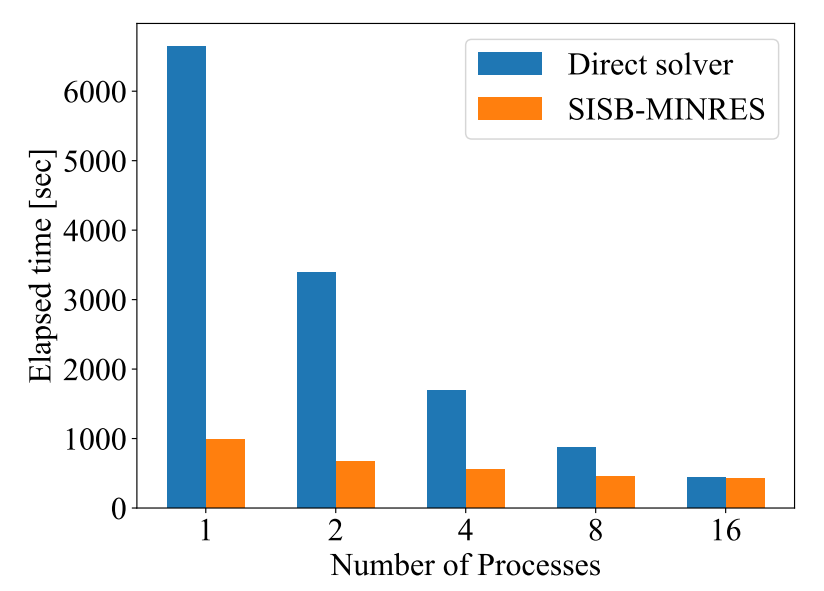

Figure 6: Elapsed time comparison for CQSZ200K.

the (scaled) residual norms:

$$
\|A \boldsymbol{u}-\lambda B \boldsymbol{u}\|_{2} /\left(\|A \boldsymbol{u}\|_{2}+\|\lambda B \boldsymbol{u}\|_{2}\right)
$$

The maximum (minimum in brackets) of the residual norms corresponding to the eigenvalues in $[a, b]$ are shown.

We find that the accuracy of eigenpairs get worse by using SISBMINRES. This may due to loss of orthogonality of the block Lanczos basis. Though the accuracy could be improved by iterating the CI eigensolver, we leave coping with this accuracy loss as our future work.

\section{CONCLUSION}

In this paper, a new approach for solving linear systems arising in the algorithm of CI eigensolvers was proposed. The proposed approach utilizes a newly presented iterative linear solver based on block Krylov subspace and shift-invert preconditioning. Based on the preconditioning, the linear solver can take advantage of the

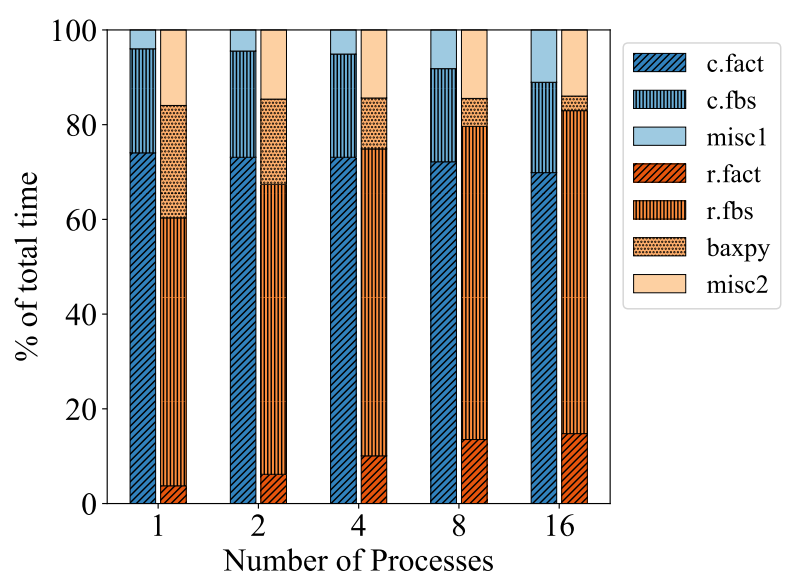

Figure 7: Breakdown of the timing for NCCS430080.

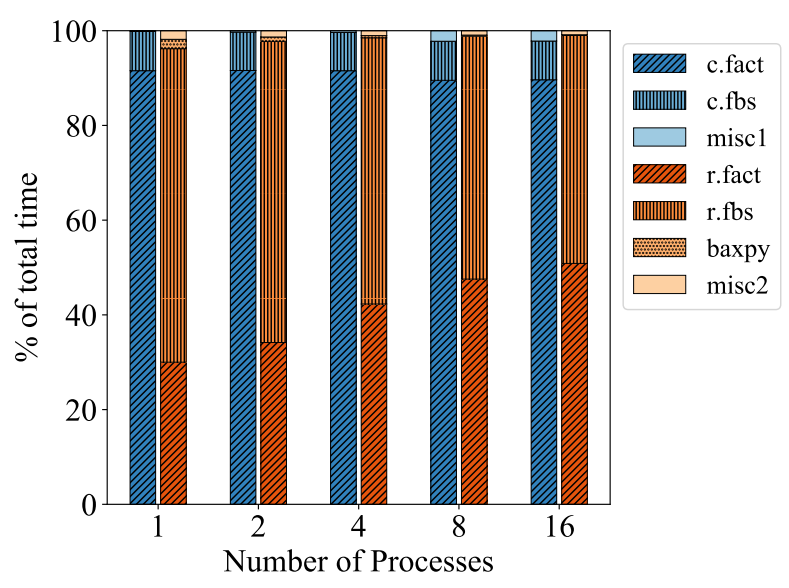

Figure 8: Breakdown of the timing for Ga41As41H72.

Table 5: Maximum (minimum) residual norms of eigenpairs.

\begin{tabular}{lrr}
\hline & Direct solver & SISB-MINRES \\
\hline NCCS430080 & $4 \mathrm{e}-11(1 \mathrm{e}-13)$ & $5 \mathrm{e}-10(2 \mathrm{e}-12)$ \\
bone010 & $3 \mathrm{e}-11(2 \mathrm{e}-12)$ & $9 \mathrm{e}-08(6 \mathrm{e}-10)$ \\
Ga41As41H72 & $2 \mathrm{e}-09(8 \mathrm{e}-13)$ & $4 \mathrm{e}-09(2 \mathrm{e}-12)$ \\
CQSZ200K & $2 \mathrm{e}-12(3 \mathrm{e}-13)$ & $3 \mathrm{e}-08(7 \mathrm{e}-11)$ \\
\hline
\end{tabular}

shift-invariance of block Krylov subspace to efficiently solve the parameterized linear systems of the CI eigensolver. Our approach adaptively sets a parameter of the preconditioning to decrease the iteration counts as the number of parallel processes increase.

In several numerical examples, our approach outperformed the direct solver approach in most cases, with some amount of accuracy loss. 
As future work, we will investigate possible approaches to overcome the accuracy loss. Additionally, we will evaluate the performance of our method using a distributed parallel direct linear solver for its capability of leveraging further degree of parallelism.

\section{ACKNOWLEDGMENTS}

This study is partially supported by the Japan Society for the Promotion of Science (JSPS), Grants-in-Aid for Scientific Research (Nos. 18H03250 and 19K20280), and Multidisciplinary Cooperative Research Program in CCS, University of Tsukuba.

\section{REFERENCES}

[1] [n.d.]. CONQUEST: Linear Scaling DFT. http://www.order-n.org/.

[2] [n.d.]. ELSES matrix library. http://www.elses.jp/matrix/.

[3] [n.d.]. FEAST Eigenvalue Solver. http://www.ecs.umass.edu/ polizzi/feast/.

[4] [n.d.]. MUMPS: MUltifrontal Massively Parallel sparse direct Solver. http:// mumps.enseeiht.fr/.

[5] [n.d.]. z-Pares: Parallel Eigenvalue Solver. https://zpares.cs.tsukubaa.ac.jp.

[6] Timothy A. Davis and Yifan Hu. 2011. The University of Florida Sparse Matrix Collection. ACM Trans. Math. Softw. 38, 1, Article 1 (Dec. 2011), 25 pages. https: //doi.org/10.1145/2049662.2049663

[7] Yasunori Futamura, Tetsuya Sakurai, Shinnosuke Furuya, and Jun-Ichi Iwata. 2013 Efficient Algorithm for Linear Systems Arising in Solutions of Eigenproblems and Its Application to Electronic-Structure Calculations. In High Performance Computing for Computational Science - VECPAR 2012, Michel Daydé, Osni Marques, and Kengo Nakajima (Eds.). Lecture Notes in Computer Science, Vol. 7851. Springer Berlin Heidelberg, 226-235. https://doi.org/10.1007/978-3-642-38718-0_23

[8] M.J. Gillan, D.R. Bowler, A.S. Torralba, and T. Miyazaki. 2007. Order-N firstprinciples calculations with the conquest code. Computer Physics Communications 177, 1 (2007), 14-18. https://doi.org/10.1016/j.cpc.2007.02.075 Proceedings of the Conference on Computational Physics 2006.

[9] Gene H. Golub and Charles F. Van Loan. 2013. Matrix Computations (4th ed.) Johns Hopkins University Press, Baltimore, MD.

[10] Roger G. Grimes, John G. Lewis, and Horst D. Simon. 1994. A Shifted Block Lanczos Algorithm for Solving Sparse Symmetric Generalized Eigenproblems. SIAM F. Matrix Anal. Appl. 15, 1 (1994), 228-272. https://doi.org/10.1137/ S0895479888151111

[11] Martin H. Gutknecht and Thomas Schmelzer. 2009. The block grade of a block Krylov space. Linear Algebra Appl. 430, 1 (2009), 174-185. https://doi.org/10. 1016/j.laa.2008.07.008

[12] Takeo Hoshi, Hiroto Imachi, Akiyoshi Kuwata, Kohsuke Kakuda, Takatoshi Fujita, and Hiroyuki Matsui. 2019. Numerical aspect of large-scale electronic state calculation for flexible device material. Japan fournal of Industrial and Applied Mathematics 36 (2019), 685-698. https://doi.org/10.1007/s13160-019-00358-2

[13] Takanori Ide, Yuto Inoue, Yasunori Futamura, and Tetsuya Sakurai. 2017. Highly Parallel Computation of Generalized Eigenvalue Problem in Vibration for Automatic Transmission of Vehicles Using the Sakurai-Sugiura Method and Supercomputers. In Mathematical Analysis of Continuum Mechanics and Industrial Applications, Hiromichi Itou, Masato Kimura, Vladimír Chalupecký, Kohji Ohtsuka, Daisuke Tagami, and Akira Takada (Eds.). Springer Singapore, Singapore, 207-218.

[14] Tsutomu Ikegami and Tetsuya Sakurai. 2010. Contour integral eigensolver for non-Hermitian systems: a Rayleigh-Ritz-type approach. Taiwanese f. Math. 14 (2010), 825-837.

[15] Tsutomu Ikegami, Tetsuya Sakurai, and Umpei Nagashima. 2010. A Filter Diagonalization for Generalized Eigenvalue Problems Based on the SakuraiSugiura Projection Method. 7. Comput. Appl. Math. 233, 8 (Feb. 2010), 1927-1936. https://doi.org/10.1016/j.cam.2009.09.029

[16] Akira Imakura, Lei Du, and Tetsuya Sakurai. 2014. A block Arnoldi-type contour integral spectral projection method for solving generalized eigenvalue problems. Applied Mathematics Letters 32 (2014), 22-27. https://doi.org/10.1016/j.aml.2014. 02.007

[17] Akira Imakura, Lei Du, and Hiroto Tadano. 2013. A Weighted Block GMRES method for solving linear systems with multiple right-hand sides. FSIAM Letters 5 (2013), 65-68. https://doi.org/10.14495/jsiaml.5.65

[18] Akira Imakura and Tetsuya Sakurai. 2018. Block SS-CAA: A complex momentbased parallel nonlinear eigensolver using the block communication-avoiding Arnoldi procedure. Parallel Comput. 74 (2018), 34-48. https://doi.org/10.1016/j. parco.2017.11.007 Parallel Matrix Algorithms and Applications (PMAA'16).

[19] J. Kestyn, V. Kalantzis, E. Polizzi, and Y. Saad. 2016. PFEAST: A High Performance Sparse Eigenvalue Solver Using Distributed-Memory Linear Solvers. In SC '16: Proceedings of the International Conference for High Performance Computing, Networking, Storage and Analysis. 178-189.
[20] Karl Meerbergen. 2003. The Solution of Parametrized Symmetric Linear Systems. SIAM 7. Matrix Anal. Appl. 24, 4 (2003), 1038-1059. https://doi.org/10.1137/ S0895479800380386

[21] Karl Meerbergen and Zhaojun Bai. 2010. The Lanczos Method for Parameterized Symmetric Linear Systems with Multiple Right-Hand Sides. SIAM 7. Matrix Anal. Appl. 31, 4 (2010), 1642-1662. https://doi.org/10.1137/08073144X

[22] Ayako Nakata, Jack S. Baker, Shereif Y. Mujahed, Jack T. L. Poulton, Sergiu Arapan, Jianbo Lin, Zamaan Raza, Sushma Yadav, Lionel Truflandier, Tsuyoshi Miyazaki, and David R. Bowler. 2020. Large scale and linear scaling DFT with the CONQUEST code. The fournal of Chemical Physics 152, 16 (2020), 164112. https://doi.org/10.1063/5.0005074

[23] Ayako Nakata, Yasunori Futamura, Tetsuya Sakurai, David R Bowler, and Tsuyoshi Miyazaki. 2017. Efficient Calculation of Electronic Structure Using $\mathrm{O}(\mathrm{N})$ Density Functional Theory. Fournal of Chemical Theory and Computation 13, 9 (2017), 4146-4153. https://doi.org/10.1021/acs.jctc.7b00385

[24] Dianne P. O'Leary. 1980. The block conjugate gradient algorithm and related methods. Linear Algebra Appl. 29 (1980), 293-322. https://doi.org/10.1016/00243795(80)90247-5 Special Volume Dedicated to Alson S. Householder.

[25] C. C. Paige and M. A. Saunders. 1975. Solution of Sparse Indefinite Systems of Linear Equations. SIAM 7. Numer. Anal. 12, 4 (1975), 617-629. https://doi.org/10. $1137 / 0712047$

[26] Eric Polizzi. 2009. Density-matrix-based algorithm for solving eigenvalue problems. Phys. Rev. B 79 (2009), 115112. Issue 11. https://doi.org/10.1103/PhysRevB. 79.115112

[27] Yousef Saad. 2011. Numerical Methods for Large Eigenvalue Problems (revised ed.). Society for Industrial and Applied Mathematics. https://doi.org/10.1137/1. 9781611970739

[28] Youcef Saad and Martin H. Schultz. 1986. GMRES: A Generalized Minimal Residual Algorithm for Solving Nonsymmetric Linear Systems. SIAM 7. Sci. Statist. Comput. 7, 3 (1986), 856-869.

[29] Tetsuya Sakurai and Hiroshi Sugiura. 2003. A projection method for generalized eigenvalue problems using numerical integration. f. Comput. Appl. Math. 159, 1 (2003), 119-128.

[30] Tetsuya Sakurai and Hiroto Tadano. 2007. CIRR: a Rayleigh-Ritz type method with contour integral for generalized eigenvalue problems. Hokkaido Math. F. 36 (2007), 745-757.

[31] Tetsuya Sakurai, Hiroto Tadano, and Yasunori Futamura. 2013. Efficient parameter estimation and implementation of a contour integral-based eigensolver. F. Algo. Comput. Tech. 7 (2013), 249-269.

[32] Olaf Schenk and Klaus Gärtner. 2011. PARDISO. In Encyclopedia of Parallel Computing, David Padua (Ed.). Springer US, Boston, MA, 1458-1464. https: //doi.org/10.1007/978-0-387-09766-4_90

[33] Kirk M. Soodhalter. 2015. A block MINRES algorithm based on the band Lanczos method. Numerical Algorithms 69, 3 (2015), 473-494. https://doi.org/10.1007/ s11075-014-9907-z

[34] Kirk M. Soodhalter. 2016. Block Krylov Subspace Recycling for Shifted Systems with Unrelated Right-Hand Sides. SIAM fournal on Scientific Computing 38, 1 (2016), A302-A324. https://doi.org/10.1137/140998214

[35] Brigitte Vital. 1990. Étude de quelques méthodes de résolution de problèmes linéaires de grande taille sur multiprocesseur. Ph. D. thesis, Université de Rennes I (1990).

[36] Yusaku Yamamoto, Yuji Nakatsukasa, Yuka Yanagisawa, and Takeshi Fukaya. 2016. Roundoff error analysis of the CholeskyQR2 algorithm in an oblique inner product. FSIAM Letters 8 (2016), 5-8. https://doi.org/10.14495/jsiaml.8.5 